\title{
Linear Stability of a Thin Film Flowing Down an Uneven Incline
}

\author{
H. A. Jasmine \\ Department of Mathematics, University of Rajshahi, \\ Rajshahi-6205, Bangladesh
}

\begin{abstract}
The linear stability of a thin film of viscous fluid falling down an uneven inclined heated wall is examined. Long wave theory developed by Benny [1] is employed. It is demonstrated that Marangoni effect makes the flow unstable when either the inclination is quite steep or when the bottom undulations are sufficiently short.
\end{abstract}

\section{Introduction}

The thin liquid film has a vast number of important industrial applications, such as cooling of thin liquid film radiator, hot fuel surfaces in nuclear reactors, rocket engines, microelectronics arrays, and gas turbine blade tips. If the fluid layer is sufficiently thin, thermocapillary convection induced by gradients in surface tension, is expected to be the dominant instability mechanism. This is known as the Marangoni effect.

Using a long wave perturbation method, Benny [1] was the first to obtain a single equation of the evolution type for the free surface in the case of isothermal flow. In the investigation of thermocapillary force on the span of supercritical/subcritical regions, Mukhopadhyay and Mukhopadhyay [2] reported that the unconditional stable region vanishes after a cutoff Marangoni number, whereas other regions increase with the increase in Marangoni number for fixed values of other parameters. Furthermore, both amplitude and nonlinear wave speed increase in the supercritical region. On the other hand, in the subcritical region, the threshold amplitude decreases with the increase in Marangoni number. By taking a large $\mathrm{Pe}^{\prime}$ clet number for the dynamics of a film going down a heated wall, Trevelyan et al. [3] show two types of wall boundary conditions: heat flux and specified temperature (ST). They employ a weighted residual attempt via a first order in $\varepsilon$ single mode Galerkin approximation of the momentum equation to develop averaged models. The heat transport was represented by two approaches: (i) a simple first order in $\varepsilon$ weighted residual approximation through a single test function which does not meet all boundary 
conditions, (ii) a refined treatment of the energy equation with a first order in $\varepsilon$ Galerkin approximation based on a set of test functions that satisfy all boundary conditions. They also formulated a long wave equation (LWE) approximation to make sure that the models obtained from their refined weigthed residuals approach based on a high-order Galerkin projection yield LWE with an appropriate gradient expansion, thus confirming the validity of their models close to criticality.

The stability of flow over uneven topography was considered by DavalosOrozco [4] who utilized a Benny type equation to analyze Newtonian flow down a vertical wall with smooth corrugations. D'Alessio et al. [5] derive and implement a model for laminar flow over an inclined surface exhibiting periodic undulations in the case of isothermal flow. Their model includes inertial and surface tension effects as well as important second-order diffusive terms by using weighted residual method. The stability of a viscous film sliding a vertical nonuniformly heated wall under gravity was studied by Samanta [6]. She shows that as $\mathrm{Pe}^{\prime}$ clet number increases, the subcritical stable region decreases while the supercritical unstable region increases.

The present investigation uses long wave theory to investigate the linear stability of a thin film falling down an uneven inclined heated wall including inertial as well as surface tension effects coupled with important second-order diffusive terms.

\section{Mathematical Formulation}

We consider the two dimensional flow of a thin viscous liquid film flowing down an uneven inclined heated plane. We define an $(\mathrm{x}, \mathrm{z})$ coordinate system with the $\mathrm{x}$-axis inclined at an angle $\beta$ with respect to the horizontal direction, and with the z-axis pointing in the upward normal direction. The surface over which the fluid is flowing is given by $\mathrm{z}=\mathrm{b}(\mathrm{x})$. The governing equations are: continuity, momentum of the fluid flow and energy for the temperature field

$$
\begin{aligned}
& \nabla \cdot \vec{u}=0, \\
& \vec{u}_{t}+(\vec{u} . \nabla) \vec{u}=-\frac{1}{\rho} \nabla p+v \nabla^{2} \vec{u}+g, \\
& T_{t}+(\vec{u} . \nabla) T=\kappa \nabla^{2} T,
\end{aligned}
$$


where $\vec{u}, \rho, \mathrm{p}$, and $\mathrm{T}$ are the velocity, density, pressure and temperature of the liquid film respectively, $\vec{g}$ is the acceleration due to gravity, $v=\mu / \rho$ is the the

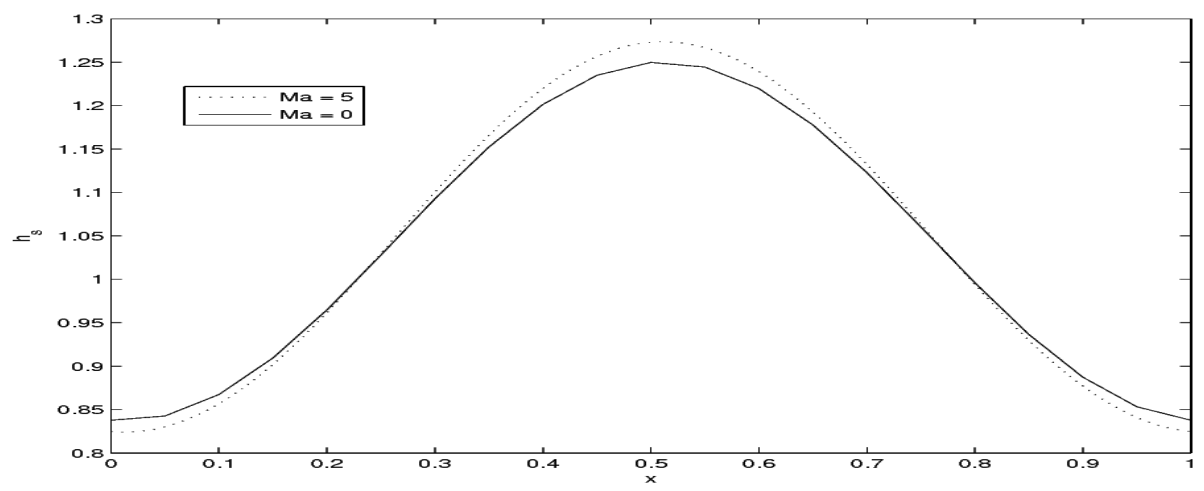

Fig.1. Numerical steady-state solution for $\mathrm{M}_{\mathrm{a}}=0,5$, and $\mathrm{W}_{\mathrm{e}}=100, \mathrm{R}_{\mathrm{e}}=5, \mathrm{a}_{\mathrm{m}}=0.2$, $\mathrm{k}=2 \pi, \cot \beta=0.5$.

kinematic viscosity, $\kappa$ is the thermal diffusivity of the liquid and $\mu$ is the magnetic permeability. On the wall, the boundary condition for the velocity is

$\mathrm{u}=\mathrm{w}=0 \quad$ on $\mathrm{z}=\mathrm{b}(\mathrm{x})$.

The thermal boundary condition for the specified temperature is

$\mathrm{T}=\mathrm{T}_{\mathrm{w}} \quad$ on $\mathrm{z}=\mathrm{b}(\mathrm{x})$,

where $T_{w}$ is the constant wall temperature for $\mathrm{ST}$. At the free surface $\mathrm{z}=\mathrm{z}_{1}=$ $\mathrm{h}(\mathrm{x}, \mathrm{t})+\mathrm{b}(\mathrm{x})$, the boundary conditions are

$\vec{n} \cdot \vec{\tau} \cdot \vec{t}=\nabla \sigma . . \vec{t}, \quad p_{a}+\vec{n} \cdot \vec{\tau} \cdot \vec{n}=\sigma(T) \nabla \cdot \vec{t}$

$h_{t}+\vec{u} \cdot \nabla(h-z)=0$,

$\lambda \nabla T \vec{n}+\alpha_{g}\left(T-T_{a}\right)=0$ 


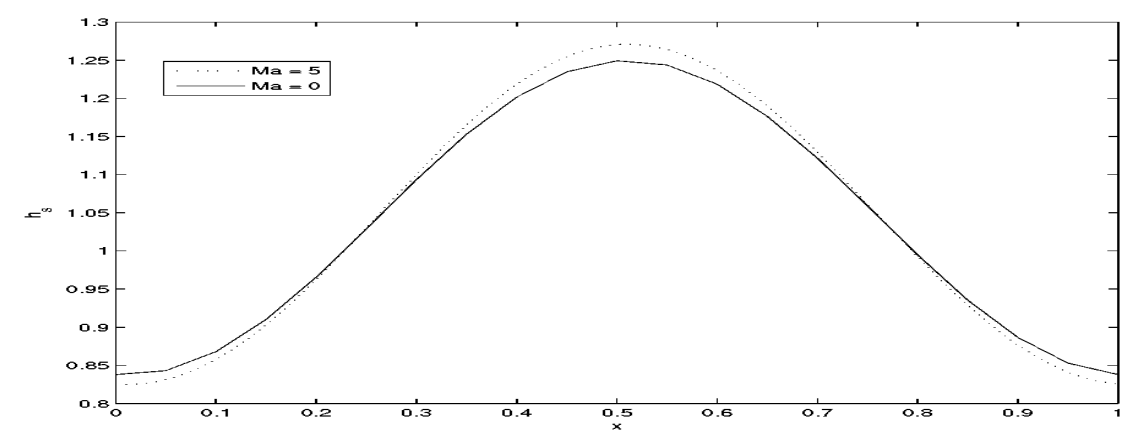

Fig.2. Numerical steady-state solution for $\mathrm{M}_{\mathrm{a}}=0,5$, and $\mathrm{W}_{\mathrm{e}}=100, \mathrm{R}_{\mathrm{e}}=5$, $\mathrm{a}_{\mathrm{m}}=0.2, \quad \mathrm{k}=2 \pi, \cot \beta=5$.

Here $\vec{n}=\frac{1}{\left(1+z_{1 x}^{2}\right)^{1 / 2}}\left[-z_{1 x, 1}\right]^{T}$ and $\vec{t}=\frac{1}{\left(1+z_{1 x}^{2}\right)^{1 / 2}}\left[1, z_{1 x}\right]$ are unit vectors normal (outward pointing) and tangential to the interface, respectively, and $\tau=-p I+2 \mu e$ is the stress tensor with the rate of strain tensor $\stackrel{e}{=}=\frac{1}{2}\left(\frac{\partial u_{i}}{\partial x_{i}}+\frac{\partial u_{j}}{\partial x_{i}}\right)$, I is the identity matrix, $\sigma$ is the surface tension of the liquid, $\mathrm{p}_{\mathrm{a}}$ is the pressure of the ambient gas phase and $\alpha_{\mathrm{g}}$ is the heat transfer co-efficient between the liquid and the air.

We have assumed the thermocapillary effect to be modeled by using a linear a approximation for the surface tension

$\sigma(T)=\sigma_{0}-\gamma\left(T-T_{a}\right)$

where $\sigma_{0}$ is the surface tension at the reference temperature, $T_{a}$ taken to be the 


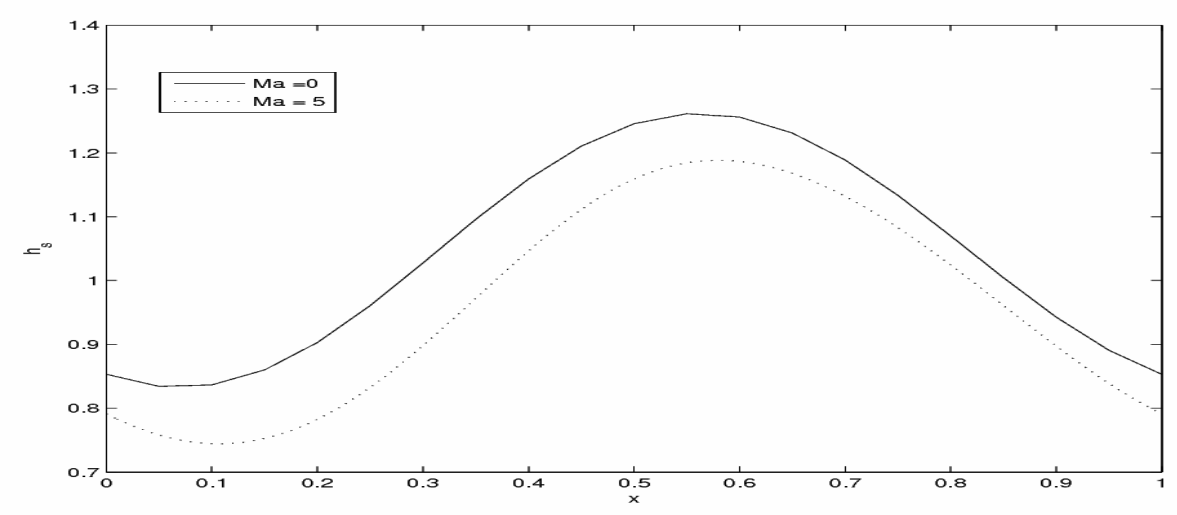

Fig.3. Numerical steady-state solution for $\mathrm{M}_{\mathrm{a}}=0,5$, and $\mathrm{W}_{\mathrm{e}}=20, \mathrm{R}_{\mathrm{e}}=5$, $\mathrm{a}_{\mathrm{m}}=0.2, \quad \mathrm{k}=2 \pi, \cot \beta=0.5$.

temperature of the ambient gas phase, and $\gamma=-\left(\frac{\partial \sigma}{\partial T}\right)_{T=T_{0}}$ is a positive constant for most common fluids.

We define the dimensionless quantities for the governing equations and boundary conditions as

$\mathrm{U}=\mathrm{Uu}^{*}, w=\frac{H}{L} U w^{*}, \quad t=\frac{L}{U} t^{*}, \mathrm{p}=\rho \mathrm{U}^{2} \mathrm{p}^{*}$,

$\mathrm{x}=\mathrm{L} \mathrm{X}, \mathrm{z}=\mathrm{Hz}^{*}, \mathrm{~T}-\mathrm{T}_{\mathrm{a}}=\Delta T T^{*}, \mathrm{~h}=\mathrm{Hh}^{*}$

The equations of motion and energy in dimensionless form become(after droping *'s)

$$
\begin{aligned}
& u_{X}+w_{z}=0 \\
& \delta R_{e}\left(u_{t}+u u_{x}+w u_{z}\right)=-\delta R_{e} p_{x}+3+\delta^{2} u_{x X}+u_{z z}
\end{aligned}
$$




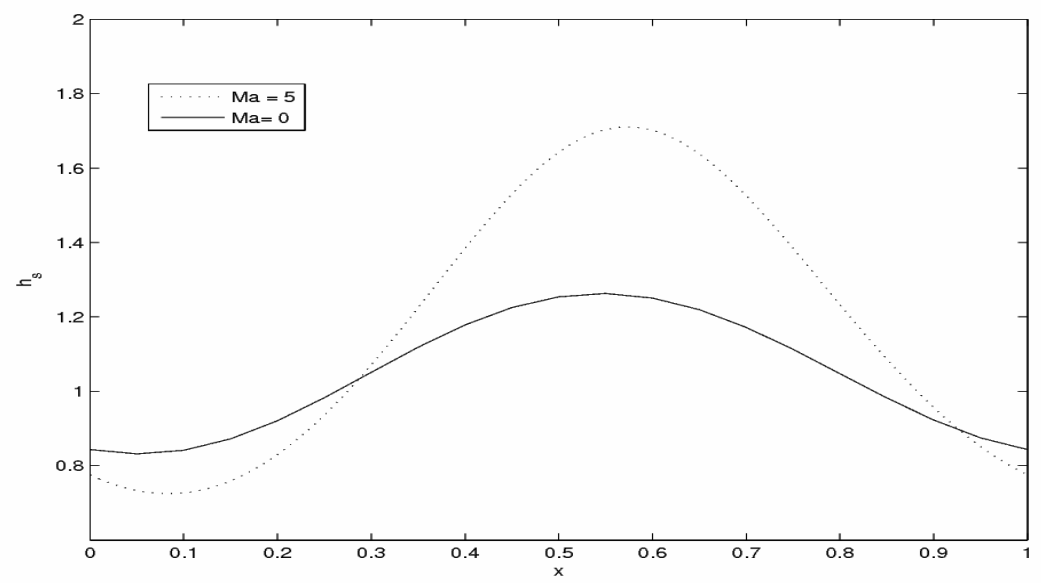

Fig.4. Numerical steady-state solution for $\mathrm{M}_{\mathrm{a}}=0,5$, and $\mathrm{W}_{\mathrm{e}}=20, \mathrm{R}_{\mathrm{e}}=5$,

$$
\mathrm{a}_{\mathrm{m}}=0.2, \mathrm{k}=2 \pi, \cot \beta=5 \text {. }
$$

$$
\delta R_{e}\left(w_{t}+u w_{X}+w w_{z}\right)=-R_{e} p_{z}-3 \cot \beta+\delta^{3} w_{x X}+\delta w_{z z}
$$

$$
\delta R_{e}\left(T_{t}+u T_{X}+w T_{Z}\right)=\frac{1}{p_{r}}\left(\delta^{2} T_{X X}+T_{z Z}\right) \text {, }
$$

where $\mathrm{U}=(\mathrm{Q} / \mathrm{H})$, Nusselt thickness $\mathrm{H}=(3 \mu \mathrm{Q} / \rho \mathrm{g} \sin \beta)^{1 / 3}$, Q is the volume flux of the equilibrium flow, $\delta=\mathrm{H} / \mathrm{L}=\mathrm{H} / \lambda_{\mathrm{b}}$ is the shallowness parameter,

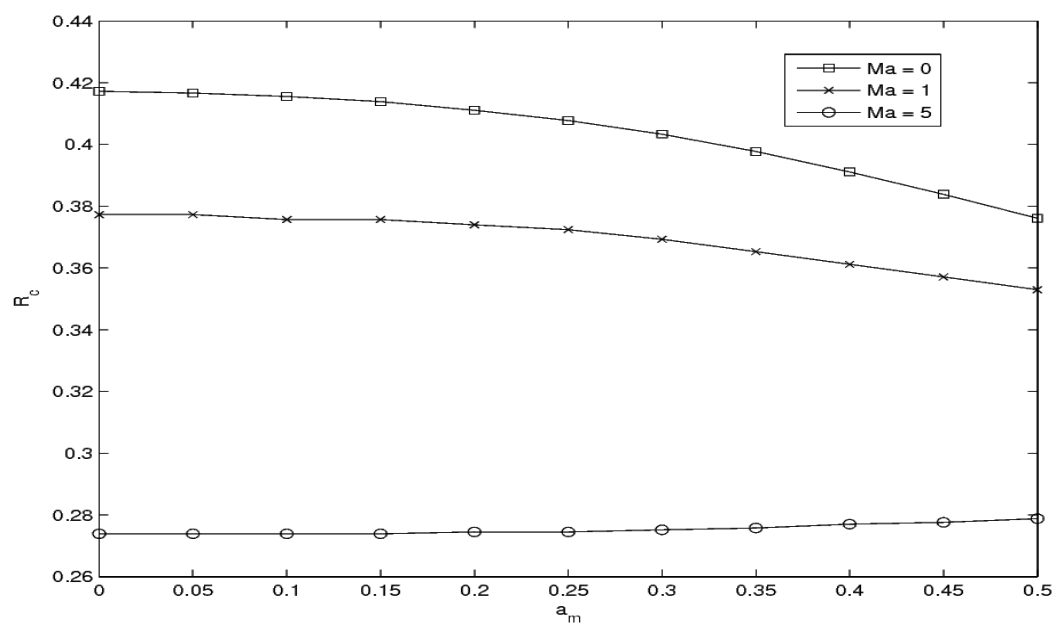

Fig. 5. Critical Reynolds number $R_{e}$ as a function of bottom amplitude $a_{m}$ for different values of Marangoni number, when $\mathrm{W}_{\mathrm{e}}=100, \delta=0.1, \mathrm{~B}=1$, and $\cot \beta=0.5$ 
$R_{\mathrm{e}}=\rho Q / \mu$ is the Reynolds number, $\mathrm{p}_{\mathrm{r}}=\nu / \kappa$ is the Prandtl number, $\Delta \mathrm{T}=\mathrm{T}_{\mathrm{W}}-\mathrm{T}_{\mathrm{a}}$, and $\lambda_{\mathrm{b}}$ is the wavelength of the bottom.

The boundary conditions at the wall $\mathrm{z}=\mathrm{b}(\mathrm{x})$ reduce to

$\mathrm{u}=\mathrm{w}=0, \quad \mathrm{~T}=1 \quad$ on $\quad \mathrm{z}=\mathrm{b}(\mathrm{x})$.

The boundary conditions at the free surface $\mathrm{z}=\mathrm{z}_{1}=\mathrm{h}(\mathrm{x}, \mathrm{t})+\mathrm{b}(\mathrm{x})$ are

$$
\begin{aligned}
& w=h_{t}+z_{1 x} u \\
& p-\frac{2}{R_{e}} \delta\left(1+\delta^{2} z_{1 x}^{2}\right)^{-1}\left(\delta^{2} z_{1 x}^{2} u_{x}+w_{z}-z_{1 x}\left(u_{z}+\delta^{2} w_{z}\right)\right)=-\left(w_{e}-M_{a} T\right) \delta^{2} z_{1 x x}\left(1+\delta^{2} z_{1 x}^{2}\right)^{-\frac{3}{2}}, \\
& \left(1-\delta^{2} z_{1 x}^{2}\right)\left(u_{z}+\delta^{2} w_{X}\right)-4 \delta^{2} z_{1 x} u_{X}=-\delta M_{a} R_{e}\left(T_{X}+z_{1 x} T_{z}\right)\left(1+\delta^{2} z_{1 x}^{2}\right)^{-\frac{1}{2}} \\
& \left(T_{z}-\delta^{2} z_{1 x} T_{X}\right)\left(1+\delta^{2} z_{1 x}^{2}\right)^{-\frac{1}{2}}+B T=0
\end{aligned}
$$

where $\mathrm{W}_{\mathrm{e}}=\sigma_{0} / \rho \mathrm{U}^{2}$ is the Weber number, $\mathrm{M}_{\mathrm{a}}=\gamma \Delta \mathrm{T} / \rho \mathrm{HU}^{2}$ is the Marangoni number, and $\mathrm{B}=\alpha_{\mathrm{g}} \mathrm{H} / \lambda_{\mathrm{b}}=\alpha_{\mathrm{g}} \mathrm{H} / \mathrm{L}$ is the Biot number.

If the Reynolds number $\mathrm{R}_{\mathrm{e}}$, Pe'clet number $\mathrm{P}_{\mathrm{e}}\left(=\mathrm{R}_{\mathrm{e}} \mathrm{P}_{\mathrm{r}}\right)$, and Marangoni number $\mathrm{M}_{\mathrm{a}}$ are assumed to be of $\mathrm{O}(1)$, and terms of $\mathrm{O}\left(\delta^{3}\right)$ and higher are neglected, then we obtain the second-order approximation to the Navier-Stokes and energy equations with respect to $\delta$ :

$$
\begin{aligned}
& u_{X}+w_{z}=0 \\
& \delta R_{e}\left(u_{t}+u u_{X}+w u_{z}\right)=-\delta R_{e} p_{X}+3+\delta^{2} u_{X X}+u_{z z}, \\
& 0=-R_{e} p_{z}-3 \cot \beta+\delta w_{z Z} \\
& \delta P_{e}\left(T_{t}+u T_{X}+w T_{z}\right)=\delta^{2} T_{X X}+T_{z z}
\end{aligned}
$$

We note that the surface tension is of second order or larger if the Weber number is of $\mathrm{O}(1 / \delta)$ or larger. Neglecting $\mathrm{O}\left(\delta^{3}\right)$ and higher order terms, the boundary conditions on $\mathrm{z}=\mathrm{b}(\mathrm{x})$ are

$\mathrm{u}=\mathrm{w}=0, \quad \mathrm{~T}=1$,

and the boundary conditions at the free surface $\mathrm{z}=\mathrm{z}_{1}=\mathrm{h}(\mathrm{x}, \mathrm{t})+\mathrm{b}(\mathrm{x})$ as 


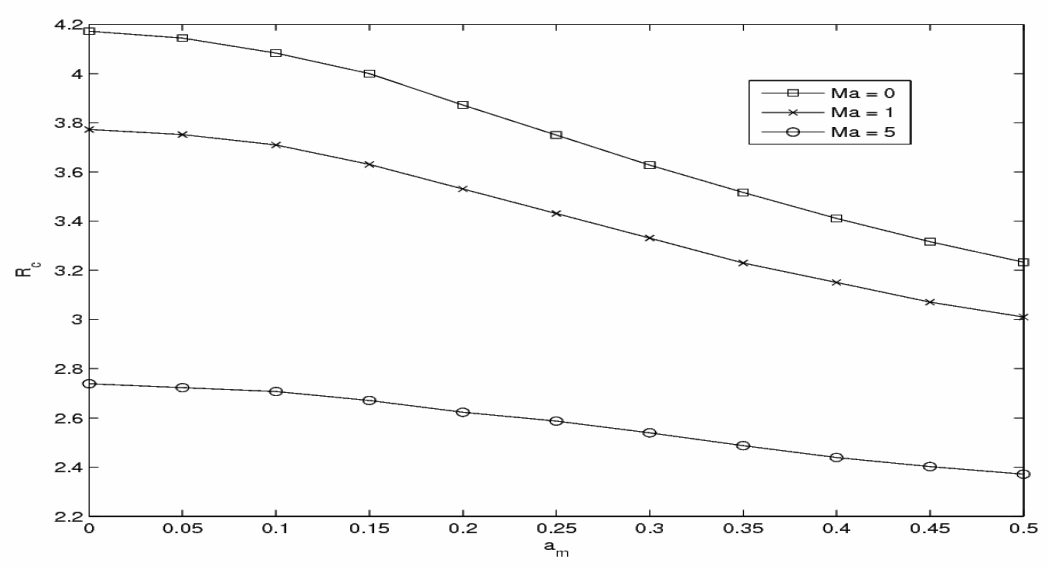

Fig. 6. Critical Reynolds number $\mathrm{R}_{\mathrm{e}}$ as a function of bottom amplitude $\mathrm{a}_{\mathrm{m}}$ for different values of Marangoni number, when $\mathrm{W}_{\mathrm{e}}=100, \delta=0.1, \mathrm{~B}=1$, and $\cot \beta=5$.

$w=h_{t}+z_{1 x} u$,

$p-\frac{2 \delta}{R_{e}} w_{z}+\delta^{2}\left(W_{e}-M_{a} T\right) z_{1 x x}=0$,

$u_{z}+\delta^{2} w_{x}-4 \delta^{2} z_{1 x} u_{x}=-\delta M_{a} R_{e}\left(T_{x}+z_{1 x} T_{Z}\right)$,

$\left(T_{Z}-\delta^{2} z_{1 x} T_{X}\right)+B T\left(1+\delta^{2} z_{1 x}^{2}\right)^{-\frac{1}{2}}=0$

$7(d)$

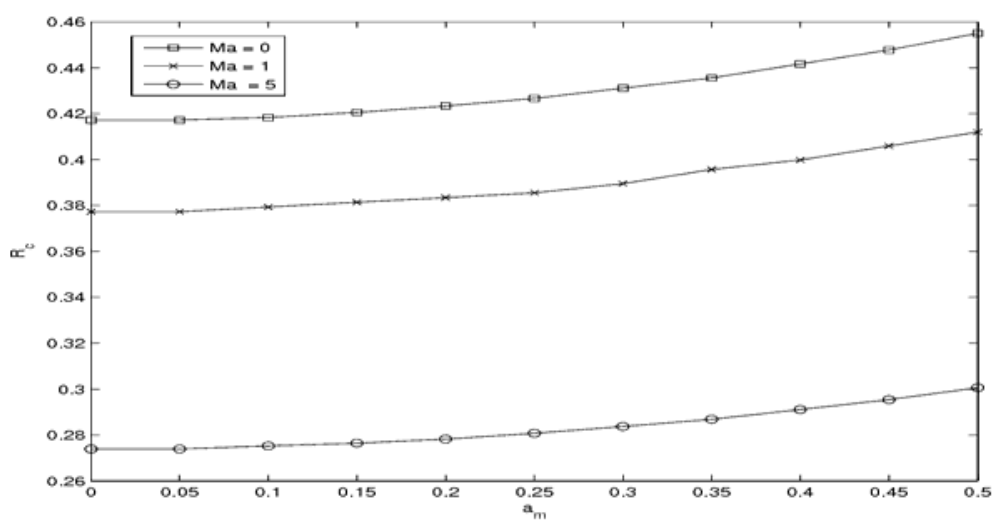

Fig. 7. Critical Reynolds number $\mathrm{R}_{\mathrm{e}}$ as a function of bottom amplitude $\mathrm{a}_{\mathrm{m}}$ for different values of Marangoni number, when $\mathrm{W}_{\mathrm{e}}=10, \delta=0.1, \mathrm{~B}=1$, and $\cot \beta=0.5$ 
The scaled bottom profile is $b(x)=a_{m} \cos (2 \pi x)$, where $a_{m}=A_{b} / H=A_{b} / \delta \lambda_{b}$ and $a_{b}=O(1)$, where the parameters $A_{b}$ and $\lambda_{b}$ the amplitude and wavelength of the undulations, respectively.

\section{Long wave approximation}

To find out the asymptotic solutions of the above system of nonlinear equations together with boundary conditions we use long wave perturbation analysis. The variables are expanded as

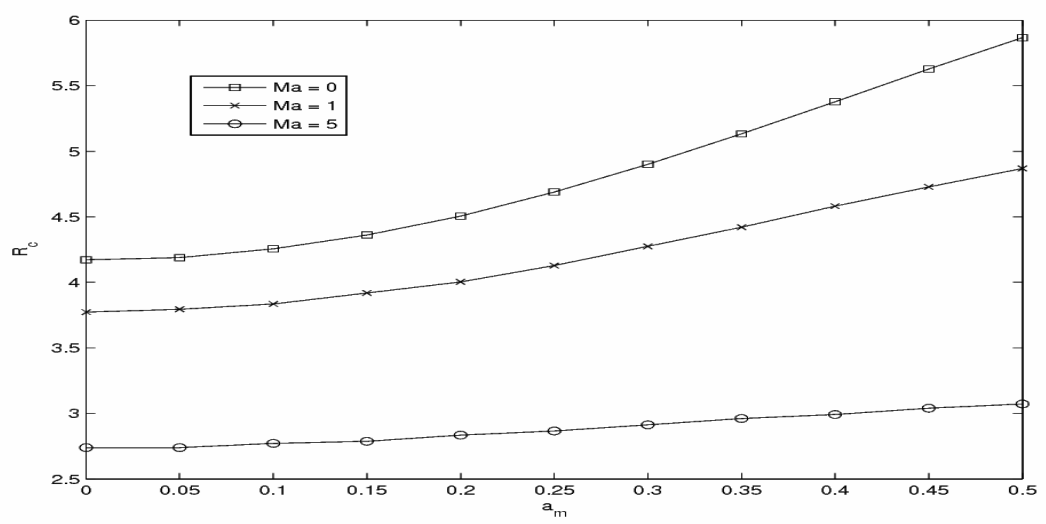

Fig. 8. Critical Reynolds number $R_{e}$ as a function of bottom amplitude $a_{m}$ for different values of Marangoni number, when $\mathrm{W}_{\mathrm{e}}=10, \delta=0.1, \mathrm{~B}=1$, and $\cot \beta=5$

$u=u_{0}+\varepsilon u_{1}+\cdots, \quad w=w_{0}+\varepsilon w_{1}+\cdots, \quad p=p_{0}+\varepsilon p_{1}+\cdots, \quad T=T_{0}+\varepsilon T_{1}+\cdots$,

These are then substituted into the system of equations (5a-5d), and boundary conditions (6a-7d). In this problem we assume $\mathrm{We} \sim \mathrm{O}\left(1 / \delta^{2}\right)$, and $\mathrm{M} \sim \mathrm{O}(1)$. The zeroth order $\left(\varepsilon^{0}\right)$ terms in equations and boundary conditions can be written as.

$u_{0 x}+w_{0 z}=0, \quad u_{0 z z}+3=0$,

$R_{e} p_{0 z}+3 \cot \beta=0, \quad T_{0 z z}=0$

Boundary conditions at $\mathrm{z}=\mathrm{b}(\mathrm{x})$ :

$\mathrm{u}_{0}=\mathrm{w}_{0}=0, \quad \mathrm{~T}_{0}=1$.

Boundary conditions at $\mathrm{z}=\mathrm{h}(\mathrm{x}, \mathrm{t})+\mathrm{b}(\mathrm{x})$ : 


$$
\begin{array}{lr}
w_{0}=h_{t}+\left(h_{x}+b_{x}\right) u_{0}, & 10(\mathrm{a}) \\
u_{0 z}=0 & 10(\mathrm{~b}) \\
p_{0}=-W_{e} \delta^{2}\left(h_{x x}+b_{x x}\right), & 10(\mathrm{c}) \\
T_{0 z}=-B T_{0} . & 10(\mathrm{~d})
\end{array}
$$

The solution of the zeroth order equations then is

$$
\begin{aligned}
& u_{0}=\frac{3}{2}(z-b)(2 h+b-z) \\
& w_{0}=\frac{3}{2}(z-b)\left[2 h b_{x}+(b-z)\left(h_{x}+b_{x}\right)\right], \\
& T_{0}=\frac{1+B(h+b-z)}{(1+B h)},
\end{aligned}
$$

and

$$
p_{0}=\frac{3 \cot \beta(h+b-z)}{R_{e}}-W_{e}\left(h_{x x}+b_{x x}\right) .
$$

The kinematic boundary condition by using zeroth order solution becomes

$$
h_{t}+3 h^{2} h_{x}=0
$$

Equating first order terms $\left(\varepsilon^{1}\right)$ from the system of equations and boundary conditions, we obtain.

$$
\begin{aligned}
& u_{1 x}+w_{1 z}=0, \\
& u_{1 z z}=R_{e} p_{0 x}+R_{e}\left(u_{0 t}+u_{0} u_{0 x}+w_{0} u_{0 z}\right), \\
& w_{0 z z}=R_{e} p_{1 z}, \quad T_{1 z z}=P_{e}\left(T_{0 t}+u_{0} T_{0 x}+w_{0} T_{0 z}\right) .
\end{aligned}
$$

Boundary conditions at $\mathrm{z}=\mathrm{b}(\mathrm{x})$

$\mathrm{u}_{1}=\mathrm{w}_{1}=0, \quad \mathrm{~T}_{1}=0$.

Boundary conditions at $\mathrm{z}=\mathrm{h}(\mathrm{x}, \mathrm{t})+\mathrm{b}(\mathrm{x})$

$w_{1}=\left(h_{x}+b_{x}\right) u_{1}$ 


$$
\begin{aligned}
& p_{1}-\frac{2}{R_{e}} w_{0 z} \\
& u_{1 z}=-M_{a} R_{e}\left[T_{0 x}+\left(h_{x}+b_{x}\right) T_{0 z}\right], \\
& T_{1 z}=-B T_{1}
\end{aligned}
$$

The solution of the first order equations then is given by

$$
\begin{aligned}
& u_{1}=\frac{1}{8}\left[(b-z)\left(R_{e} u_{11} h_{x}+R_{e} u_{12} h_{t}+u_{13}\right)\right], \\
& w_{1}=R_{e}(b-z)\left[\left(h_{x}\right)^{2} w_{11}+h_{x} w_{12}+h_{x x} w_{13}+h_{t} h_{x} w_{14}+h_{t} w_{15}+h_{x t} w_{16}+h_{x} w_{17}+w_{18}\right] \\
& p_{1}=\frac{3}{R_{e}}\left[(h+b) b_{x}-(h+z) h_{x}\right] \\
& T_{1}=\frac{P_{e} B F^{2} h_{x}}{40}\left[\left(T_{11}+T_{12}\right) z^{2}-T_{13}-F\left(T_{14}+T_{15}\right)\right],
\end{aligned}
$$

where

$$
\begin{aligned}
& u_{11}=3\left(h b^{3}-3 h b^{2} z+3 h b z^{2}-h z^{3}+4 h^{4}\right)-M_{a} B F^{2}, \\
& u_{12}=\left(8 b z-4 b^{2}+12 h^{2}-4 z^{2}\right), u_{13}=-4(z-b-2 h) p_{0 x} \cot \beta, \quad F=\frac{1}{(1+B h)}, \\
& w_{11}=\frac{1}{40}\left[3(b-z)\left((b-z)^{2}+40 h^{3}\right)\right]+M_{a} B^{2} F^{2}(b-z), \\
& w_{12}=\frac{1}{8}\left[3 h b_{x}\left((b-z)^{3}+4 h^{3}\right)\right]-M_{a} B F^{2} b_{x} \\
& w_{13}=\frac{1}{40}\left[3 h(b-z)\left((b-z)^{3}+10 h^{3}\right)\right]-\frac{1}{2} M_{a} B F^{2}(b-z), \\
& w_{14}=\frac{3 h}{2}(b-z), \quad w_{15}=-\frac{1}{2} b_{x}\left[(b-z)^{2}-3 h^{2}\right], \\
& w_{16}=-\frac{1}{8}(b-z)\left[(b-z)-6 h^{2}\right], \quad w_{17}=\frac{1}{2}(b-z) p_{0 x}, \\
& w_{18}=\frac{1}{2}[b-z+2 h] s_{x} p_{0 x}+\frac{1}{6}[(b-z)(b-z+3 h)] p_{0 x x}, \\
& \left.T_{11}=-\left[3 z^{3} B-15 h B z^{2}-5 z^{2}+20 h^{2} B^{2} z\right], \quad T_{12}=\mid 15 B z^{2}-20 z-15 B h z+60 B h^{2}\right] b \\
& \left.T_{13}=\left[3 B(5 z-b) b^{2}+20 B h^{2}(3 z-b)\right] b^{2}, \quad T_{14}=-2 h^{3} \mid 4 B^{2} h^{2}+5 B h-10\right] z, \\
& T_{15}=[B h(b+4 z)(4+3 B h)+20(z-b)] b^{3}+\left|2 h^{3}\left(4 B^{2} h^{2}+5 B h-10\right)\right| b .
\end{aligned}
$$

When these values are substituted in the solutions of the zeroth order and first order equations as well as into the kinematic boundary condition (7a), we obtain $h_{t}+3 h^{2} h_{x}+\delta\left[\frac{6}{5} R_{e} h^{6} h_{x}+\frac{1}{2} M_{a} R_{e} B F^{2} h^{2} h_{x}-\cot \beta h^{3}\left(h_{x}+b_{x}\right)+\frac{\delta^{2}}{3} W_{e} R_{e} h^{3}\left(h_{x x x}+b_{x x x}\right)\right]_{x}=0$ (20) 
Equation (20) represents the first order Benny [1] type equation for the viscous fluid flowing down an uneven incline with heated wall. When $h=1$, and $b=0$, the critical condition for ST yields

$$
R_{e}=\frac{5}{6} \cot \beta-\frac{5}{12} M_{a} B F^{2},
$$

where $R_{c}$ is the critical Reynolds number. When $M_{a}>0$, the Marangoni effect is destabilized as $R_{c}$ decreases with increase in $M_{a}$. When $M_{a}<0$, the Marangoni effect is stabilizes the flow. For $M_{a}=0$, the above equation reduces to the wellknown critical condition for free film falling. That is $R_{c}=5 / 6 \cot \beta$ which is the critical $\mathrm{R}_{\mathrm{e}}$ for the isothermal case as obtained by Benjamin [7] and Yih [8]

\section{Linear Stability Analysis}

The steady-state solution to the equation (20) is given by $h=h_{s}(x)$ where $h_{s}(x)$ satisfies the nonlinear differential equation given by

$$
3 h_{s}^{2} h_{s}^{\prime}+\delta\left[\frac{6}{5} R_{e} h_{s}^{6} h_{s}^{\prime}+\frac{1}{2} M_{a} R_{e} B F^{2} h_{s}^{2} h_{s}^{\prime}-\cot \beta h_{s}^{3}\left(h^{\prime}+b^{\prime}\right)+\frac{\delta^{2}}{3} W_{e} R_{e} h_{s}^{3}\left(h_{s}^{\prime \prime \prime}+b^{\prime \prime \prime}\right)\right]^{\prime}=0
$$

where prime denotes differential with respect to $\mathrm{x}$. We have solved the equation (22) by using the MATLAB routine bvp4c. Figures 1-4 represents numerical steady-state solution when $\mathrm{M}_{\mathrm{a}}=0, \mathrm{M}_{\mathrm{a}}=5, \mathrm{R}_{\mathrm{e}}=5, \mathrm{a}_{\mathrm{m}}=0.2, \mathrm{k}=2 \pi$, and $\mathrm{B}=1$. In figure $1, \mathrm{~W}_{\mathrm{e}}=100$ and $\cot \beta=0.5$. In figure $2, \mathrm{~W}_{\mathrm{e}}=100$ and $\cot \beta=5$. In figure 3 , $\mathrm{W}_{\mathrm{e}}=20$ and $\cot \beta=0.5$. In figure $4, \mathrm{~W}_{\mathrm{e}}=20$ and $\cot \beta=5$. All these figures show that Marangoni effect has significant influence on steady-state solution. When $\mathrm{M}_{\mathrm{a}}=0$, our result is consistent with isothermal case investigated by D'Alessio et al. [5].

We linearize the equation (20) by introducing perturbation $\eta$ and setting $h=h_{s}(x)+\varepsilon \eta_{n}(x, t)$

The linearized perturbation equations can be written in the form

$\frac{\partial \eta}{\partial t}+C_{1} \eta+C_{2} \frac{\partial \eta}{\partial x}+C_{3} \frac{\partial^{2} \eta}{\partial x^{2}}+C_{4} \frac{\partial^{3} \eta}{\partial x^{3}}+C_{5} \frac{\partial^{4} \eta}{\partial x^{4}}=0$

where 


$$
\begin{aligned}
& C_{1}=\frac{1}{5\left(1+h_{s} B\right)^{4}}\left(120 h_{s}^{4} h_{s}^{\prime} B^{3}+18 h_{s}^{3} h_{s}^{\prime} B^{2}+30 h_{s}^{5} h_{s}^{\prime} B^{4}+30 h_{s} h_{s}^{\prime}+30 \delta A h_{s}\left(h_{s}^{\prime}\right)^{2}-15 \delta R_{e} A h_{s}^{2} h_{s}^{\prime \prime}\right. \\
& +1808 R_{e} h_{s}^{4}\left(h_{s}^{\prime}\right)^{2}-15 \delta A h_{s}^{2} b^{\prime \prime}+360 R_{e} h_{s}^{5} h_{s}^{\prime \prime}+5 \delta^{3} W_{e} R_{e} h_{s}^{6} h_{s}^{\prime \prime \prime \prime} B^{4}+1800 R_{e} h_{s}^{8}\left(h_{s}^{\prime}\right)^{2} B^{4}++30 \delta^{3} W_{e} R_{e} h_{s}^{4} h_{s}^{\prime \prime \prime \prime} B^{2} \\
& +20 \delta^{3} W_{e} R_{e} h_{s}^{5} h_{s}^{\prime \prime \prime \prime} B^{3}+120 \delta A h_{s}^{4}\left(h_{s}^{\prime}\right)^{2} B^{3}+5 \delta^{3} W_{e} R_{e} h_{s}^{2} b^{\prime \prime \prime \prime}-15 \delta A h_{s}^{6} b^{\prime \prime} B^{4}+10 \delta^{3} W_{e} R_{e} h_{s}^{5} h_{s}^{\prime} h_{s}^{\prime \prime \prime} \\
& -30 \delta A h_{s}^{5}\left(h_{s}^{\prime}\right)^{2} B^{4}-10 \delta M_{a} R_{e} B^{2} h_{s}\left(h_{s}^{\prime}\right)^{2}+144 \delta R_{e} h_{s}^{6} h_{s}^{\prime \prime} B+5 \delta M_{a} R_{e} B\left(h_{s}^{\prime}\right)^{2}+5 \delta M_{a} R_{e} B h_{s} h_{s}^{\prime \prime}+720 \delta R_{e} h_{s}^{5}\left(h_{s}^{\prime}\right)^{2} B \\
& +120 \delta A h_{s}^{2}\left(h_{s}^{\prime}\right)^{2} B+5 \delta M_{a} R_{e} B^{2} h_{s}^{2} h_{s}^{\prime \prime}-120 \delta A h_{s}^{2} h_{s}^{\prime} b^{\prime} B+5 \delta^{3} W_{e} R_{e} h_{s}^{2} h_{s}^{\prime \prime \prime \prime}+20 \delta^{3} W_{e} R_{e} h_{s}^{3} h_{s}^{\prime \prime \prime \prime} B \\
& +5 \delta^{3} W_{e} R_{e} h_{s}^{6} b^{\prime \prime \prime \prime} B^{4}-15 \delta A h_{s}^{6} h_{s}^{\prime \prime} B^{4}+36 \delta R_{e} h_{s}^{9} h_{s}^{\prime \prime} B^{4}-30 \delta A h_{s}^{5} h_{s}^{\prime} b^{\prime} B^{4}+10 \delta^{3} W_{e} R_{e} h_{s}^{5} h_{s}^{\prime} b^{\prime \prime \prime} B^{4} \\
& +30 \delta^{3} W_{e} R_{e} h_{s}^{4} b^{\prime \prime \prime \prime} B^{2}+20 \delta^{3} W_{e} R_{e} h_{s}^{5} b^{\prime \prime \prime \prime} B^{3}+20 \delta^{3} W_{e} R_{e} h_{s}^{3} b^{\prime \prime \prime \prime} B+10808 R_{e} h_{s}^{6}\left(h_{s}^{\prime}\right)^{2} B^{2} \\
& -90 \delta A h_{s}^{4} b^{\prime \prime} B^{2}-60 \delta A h_{s}^{5} b^{\prime \prime} B^{3}+2168 R_{e} h_{s}^{7} h_{s}^{\prime /} B^{2}-60 \delta A h_{s}^{3} b^{\prime \prime} B+1448 R_{e} h_{s}^{8} h_{s}^{\prime /} B^{3}-180 \delta A h_{s}^{3} h_{s}^{\prime} b^{\prime} B^{2} \\
& -120 \delta A h_{s}^{4} h_{s}^{\prime} b^{\prime} B^{3}+10 \delta^{3} W_{e} R_{e} h_{s} h_{s}^{\prime} h_{s}^{\prime \prime}+40 \delta^{3} W_{e} R_{e} h_{s}^{2} h_{s}^{\prime} h_{s}^{\prime \prime \prime} B+720 \Omega R_{e} h_{s}^{7}\left(h_{s}^{\prime}\right)^{2} B^{3}-60 A h_{s}^{5} h_{s}^{\prime \prime} B^{3} \\
& -90 \delta A h_{s}^{4} h_{s}^{\prime \prime} B^{2}-60 \delta A h_{s}^{3} h_{s}^{\prime \prime} B-30 \delta A h_{s} h_{s}^{\prime} b^{\prime}-180 \delta A h_{s}^{3}\left(h_{s}^{\prime}\right)^{2} B^{2}+60 \delta^{3} W_{e} R_{e} h_{s}^{3} h_{s}^{\prime} h_{s}^{\prime \prime \prime} B^{2} \\
& +10 \delta^{3} W_{e} R_{e} h_{s} h_{s}^{\prime} b^{\prime \prime \prime}+40 \delta^{3} W_{e} R_{e} h_{s}^{2} h_{s}^{\prime} b^{\prime \prime \prime} B+60 \delta^{3} W_{e} R_{e} h_{s}^{3} h_{s}^{\prime} b^{\prime \prime \prime} B^{2}+40 \delta^{3} W_{e} R_{e} h_{s}^{4} h_{s}^{\prime} h_{s}^{\prime \prime \prime} B^{3} \\
& \left.+40 \delta^{3} W_{e} R_{e} h_{s}^{4} h_{s}^{\prime} b^{\prime \prime \prime} B^{3}+120 h_{s}^{2} h_{s}^{\prime} B\right) \\
& C_{2}=\frac{1}{5\left(1+h_{s} B\right)^{3}}\left(\left(45 h_{s}^{2} B+45 h_{s}^{3} B^{2}+15 h_{s}^{4} B^{3}+5 \delta^{3} W_{e} R_{e} h_{s}^{4} h^{\prime \prime \prime} B^{3}+15 \delta^{3} W_{e} R_{e} h_{s}^{2} b^{\prime \prime \prime} B+15 \delta^{3} W_{e} R_{e} h_{s}^{3} b^{\prime \prime \prime} B^{2}\right.\right. \\
& -45 \delta A h_{s}^{2} b^{\prime} B+2160 R_{e} h_{s}^{6} h_{s}^{\prime} B^{2}+2160 R_{e} h_{s}^{5} h^{\prime} B-15 \delta A h_{s}^{4} b^{\prime} B^{3}+15 \delta^{3} W_{e} R_{e} h_{s}^{2} h^{\prime \prime \prime} B+5 \delta^{3} W_{e} R_{e} h_{s}^{4} b^{\prime \prime \prime} B^{3}+ \\
& 5 \delta^{3} W_{e} R_{e} h_{s} h_{s}^{\prime \prime}+10 \delta M_{a} R_{e} h_{s}^{\prime} B-90 \delta A h_{s}^{2} h_{s}^{\prime} B+15 \delta^{3} W_{e} R_{e} h_{s}^{3} h_{s}^{\prime \prime \prime} B^{2}+72 \delta h_{s}^{7} h_{s}^{\prime} B^{3}+5 \delta^{3} W_{e} R_{e} h_{s} b^{\prime \prime \prime} \\
& \left.\left.-30 \delta A h_{s}^{4} h_{s}^{\prime} B^{4^{3}}-90 \delta A h_{s}^{3} h_{s}^{\prime} B^{2}-45 \delta A h_{s}^{3} b^{\prime} B^{2}+15 h_{s}+72 \delta R_{e} h_{s}^{4} h_{s}^{\prime}-30 \delta A h_{s} h_{s}^{\prime}-15 \delta A h_{s} b^{\prime}\right) h_{s}\right) \\
& C_{3}=\frac{1}{10\left(1+h_{s} B\right)^{2}}\left(\left(12 R_{e} h_{s}^{6} B^{2}-10 A h_{s}^{3} B^{2}+24 R_{e} h_{s}^{5} B-20 A h_{s}^{2} B+5 M_{a} R_{e} B-10 A h_{s}+12 R_{e} h_{s}^{4}\right) \delta h_{s}^{2}\right) \\
& C_{4}=\delta^{3} W_{e} R_{e} h_{s}^{2} h_{s}^{\prime}, \quad C_{5}=\frac{1}{3} \delta^{3} W_{e} R_{e} h_{s}^{3}, \\
& \text { where } A=\cot \beta
\end{aligned}
$$

The coefficients in equation (23) are periodic functions for the case of an uneven bottom. We conduct the stability analysis by apply Floquet-Bloch theory. We consider the perturbation as Bloch-type function in the form 
$\eta=e^{\sigma t} e^{i K x} \sum_{n=-\infty}^{\infty} \eta_{n} e^{i n k x}$

Introducing the Bloch-type functions with truncated series into the perturbation equations yields an algebraic eigenvalue problem. The exponential factor containing the bloch wave number $\mathrm{K}$ represents disturbances which interact with the periodic bottom topography via the equilibrium flow. The later is represented by the Fourier series composed of the harmonics of $\mathrm{k}$. In the case of weak bottom undulations, an approximate method of solution making use of the analytical steady-state solution can be employed. However, in the general case, the algebraic eigenvalue problem must be solved numerically for the temporal growth rate $\mathfrak{R}(\sigma)$. In this way we can determine the critical Reynolds number for the onset of instability, and for supercritical flows we can compute the wavelength and speed of unstable disturbances.

For the even bottom case, our results reveal that while the critical Reynolds number is independent of the Weber number, in the presence of bottom topography the onset of instability is strongly influenced by the effect of Marangoni number and surface tension. In figures 5-8, we present the distribution of the critical Reynolds number $R_{c}$ with the amplitude of the bottom undulation $\mathrm{a}_{\mathrm{m}}$, for several values of Marangoni number, $\mathrm{k}=2 \pi$, and $\mathrm{B}=1$. In figure $5, \mathrm{~W}_{\mathrm{e}}=100$ and $\cot \beta=0.5$. In figure $6, \mathrm{~W}_{\mathrm{e}}=100$ and $\cot \beta=5$. In figure $7, \mathrm{~W}_{\mathrm{e}}=10$ and $\cot \beta=0.5$. In figure $8, \mathrm{~W}_{\mathrm{e}}=10$ and $\cot \beta=5$. Our results show that as Marangoni effect increases with decreasing Reynolds number, the Marangoni effect is destabilizing the flow. When $M_{a}=0$, our results are consistent with those of D'Alessio et al. [5] for isothermal case.

\section{Conclusion}

We investigate the periodically varying bottom topography on the linear stability with combined effect of thermal and strong surface tension. These include a mathematical model based on the long wave theory first proposed by Benny [1] for even bottom. This model we have extended to account for heated wavy bottom topography. Numerical simulations have been done for different values of Marangoni number and surface tension. Our numerical results show that as Marangoni number increases, critical Reynolds number decreases and flow becomes unstable. Asymptotic analyses have also been conducted to both complement and extend our finding. Theoretical predictions were supported by our numerical simulations. We conclude that Marangoni effect shall destabilize the flow provided that the inclination is sufficiently steep or the bottom undulation are sufficiently short. 


\section{References}

1. Benny, D. J : J. Math. Phys., 45, 150 (1966)

2. Mukhopadhyay, A and Mukhopadhyay, A: J. Phys. D, 40, 5683 (2008)

3. Trevelyan , P. M. J, Scheid, B, Ruyer-Quil, C and Kalliadasis, S : J. Fluid Mech., 592, 295 (2007)

4. Davalos-Orozco, L. A : Phys. Fluid, 19, 074193 (2007)

5. D’Alessia, S. J. D, Pascal, J. P and Jasmine H. A : Phys. Fluid, 21, 062105 (2009)

6. Samanta, A : Physica D, 237, 2587 (2008)

7. Benjamin, T. B: J. Fluid Mech., 2, 554 (1957)

8. Yih, C. S : Phys. Fluid, $\underline{6}, 321$ (1963) 